\title{
CORPOGRAPHIES: EXPLORATIONS DU CORPS FACE AU POLITIQUE
} CORPOGRAFÍAS : EXPLORACIONES DEL CUERPO FRENTE A LO POLÍTICO

Martha Liliana Amorocho ${ }^{1}$ 


\section{Resumo}

Empruntée à la pensée de la décolonisation, la conception du corps comme territoire continue, encore aujourd'hui - et avec force, à servir de lieu de contestation pour des artistes femmes en Amérique latine. Nous le découvrons à travers l'analyse des œuvres photographiques et performatives des artistes que nous avons choisies d'étudier. Celles-ci réussissent à créer une écriture du corps aussi bien par sa fragmentation et son déchiffrement que par son utilisation comme support tant d'un imaginaire érotique que d'une prise de position politique et sociale. Les œuvres analysées composent des phrases transgressives qui transforment la matérialité du corps en signe, en décrivent les paysages intimes, en érigent des cartographies et en déjouent les frontières. Instituant l'image en prise de parole contre la violence, le féminicide, l'extractivisme et la domination en vue d'une ré-appropriation du corps, elles mettent le doigt dans le vif d'une réalité sociale encore trop présente nous concernant tous.

Palavras-chave: Corps-territoire, cartographie, extractivisme, violences de genre, féminicide.

\section{Resumen}

Tomada del pensamiento de la descolonización, la concepción del cuerpo como territorio continúa siendo utilizada, aún hoy -y con fuerza, como lugar de protesta por artistas mujeres en América Latina. Lo descubrimos a través del análisis de obras fotográficas y performativas que hemos escogido para el presente estudio. Estas artistas logran crear una escritura del cuerpo gracias a una fragmentación de este y a su desciframiento; y por otro lado, mediante su utilización como soporte tanto de un imaginario erótico como de un posicionamiento político y social. Las obras analizadas en este artículo componen frases transgresivas que transforman la materialidad del cuerpo en signo, describiendo paisajes íntimos, erigiendo cartografías y desdibujando las fronteras. En vista de una reapropiación del cuerpo, ellas instituyen la imagen como portavoz contra la violencia, el feminicidio, el extractivismo y la dominación, tocando así las llagas de una realidad social que sigue vivamente presente y que nos concierne a todos.

Palabras claves: Cuerpo-territorio, cartografía, extractivismo, violencia de género, feminicidio.

ISSN: 2175-2346

1 m_amorocho@yahoo.es 


\section{Introducion}

Empruntée à la pensée de la décolonisation, la conception du corps comme territoire continue, encore aujourd'hui - et avec force, à servir de lieu de contestation pour des artistes femmes en Amérique latine. C'est pourquoi, sous le titre « Corpographies », nous cherchons à analyser quelques œuvres plastiques contenant des analogies à " fleur de peau » et naviguant entre les notions de cartographie, de corps et de territoire. Portant un regard sur le corps en tant que géographie, nous partons en quête de métaphores y faisant référence afin d'établir les lignes directrices de notre analyse. Pour ce faire, nous nous sommes appuyés sur des rapprochements de ce genre effectués en Amérique latine tant sur le plan politique que culturel. Pour plusieurs groupes féministes en effet, le corps, spécialement celui de la femme, y est considéré comme le premier terrain à « reconquérir ». Les analogies entre corps et territoire y sont ouvertement affichées comme étendards lors des manifestations politiques (CRUZ HERNÁNDEZ, 2017). Par exemple, pour les femmes de l'Amazonie équatorienne s'opposant à l'extractivismo (l'extraction des ressources naturelles affectant les communautés et l'écosystème locaux), le corps féminin comme « territoire-corps " constitue le premier sujet à se réapproprier, permettant ensuite le passage à la défense du « territoire-terre »(GARTOR, 2014). Des artistes et des commissaires d'art font également ce rapprochement, notamment le plasticien performeur et activiste Guillermo Gómez-Peña, qui suggère que les corps colonisés de l'altérité (surtout ceux des femmes, des gays, ou des personnes « de couleur ») est le premier lieu à libérer de la domination ;

Nos corps sont aussi des territoires occupés. Peut-être que le but ultime de la performance, surtout si vous êtes une femme, un gay ou une personne "de couleur", est de décoloniser nos corps et de rendre apparent aux personnes de notre auditoire ces mécanismes de décolonisation, dans l'espoir qu'elles seront inspirées à faire de même avec le leur. (2005, p. 24. Traduction personnelle.)

Incitée par cet ensemble de parallèles, nous avons choisi quelques œuvres qui explorent des géographies corporelles de manières différentes, traçant chaque fois des cartographies particulières.

Or la carte, comme l'écrit Lucy Lippard dans son livre Overlay, « [...] est simultanément un lieu, un voyage et un concept mental ; abstrait et figuratif ; lointain et intime. Les cartes sont comme des "instantanés" d'un voyage, un arrêt sur image » (1983, p. 122). Dans les travaux artistiques choisis pour cet article, cet arrêt sur image se fera sur le corps, en premier lieu sur la peau. Mais qu'est-ce qui fait que ces artistes utilisent un référencement corporel et cartographique comme point de départ pour orienter leurs inquiétudes vers des questionnements ouvertement sociaux et politiques? Quels rapports se tressent-ils à l'intérieur des images que ces créatrices utilisent et fabriquent ? En se rapprochant du corps, en le fragmentant, en intervenant dessus, les artistes auxquelles nous ferons référence vont le « cartographier », chacune selon sa démarche, y installant ainsi une relation métaphorique et symbolique entre une réalité abstraite et sa représentation. Différentes thématiques vont ainsi être tissées tout au long de cette exploration. Nous commencerons par le corps ré-approprié qui résulte en un imaginaire érotique dans un parcours fragmenté où l'artiste se lance à la recherche d'un fantasmatique point G (Urquijo, Punto G, 1996). Élargissant notre 
point de vue de l'intime au sociétal et au politique, les travaux artistiques analysés par la suite sont des positionnements face à des problématiques sociales tels les déplacements forcés, l'extractivisme et le féminicide. La constante dans l'ensemble des œuvres présentées à partir de là est la volonté de déceler les relations de pouvoir imperceptibles agencées sur les corps.

\section{Une (dé)marche fragmentaire}

Parcourir la superficie corporelle est la démarche que l'artiste colombienne Lisette Urquijo a choisie dans la réalisation de deux œuvres: Punto G (1996) et Inventario (2003). Traçant des routes fictives avec son appareil photographique, l'artiste est partie à la recherche imaginaire de zones érogènes dans le corps d'une jeune femme, un supposé Point G. Ce travail est composé de 80 photographies argentiques en noir et blanc à une échelle de 5 × $5 \mathrm{~cm}$ chacune, formant dans leur ensemble une sorte de grand paysage cutané, une cartographie. La traversée que Lisette Urquijo enregistre est un trajet fragmentaire, un visage en entier parfois ou un œil isolé, le regard de l'artiste se focalisant sur des portions anatomiques de différentes étendues. Par ce morcellement spécifique du corps et de son ré-assemblage ultérieur, Urquijo transforme en métaphore son "voyage ". Il ne s'agit plus d'une métonymie où une portion de peau, une main ou un œil viennent signifier la totalité corporelle; mais par la manière de mettre en relation ces photos, une autre dimension vient prendre corps, celle du corps érotisé.

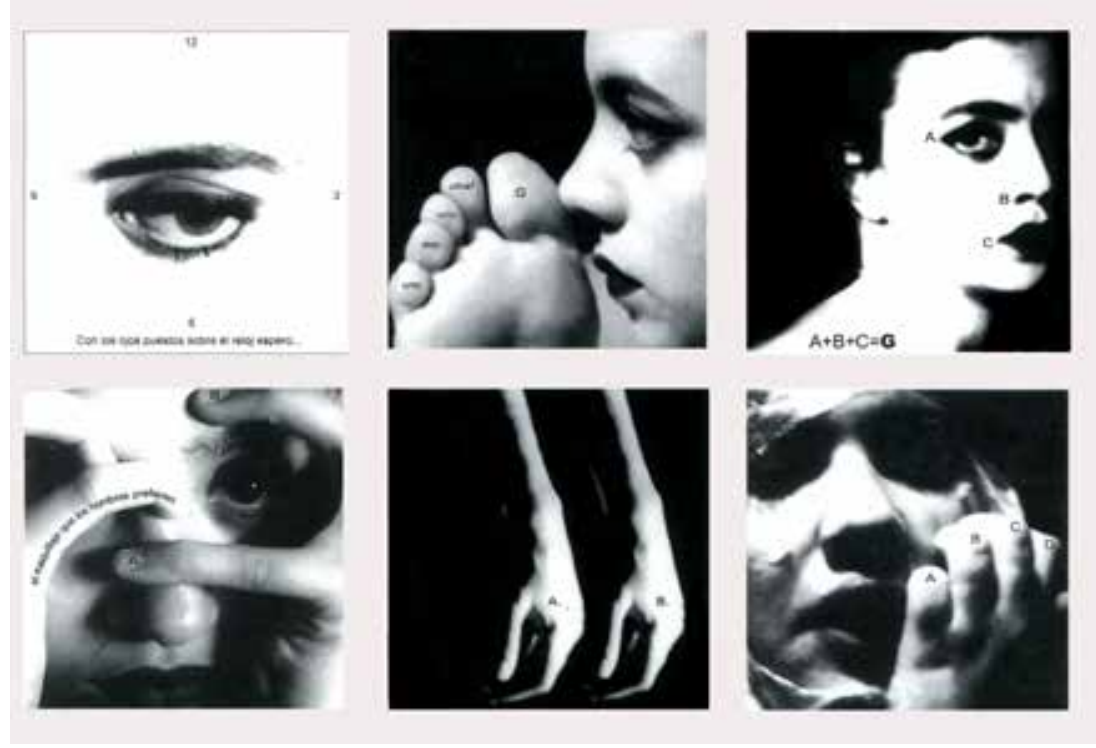

Fig. 1 - Lisette Urquijo. Punto G. 80 photographies noir et blanc.

$5 \times 5 \mathrm{~cm}$ chaque image. 1996.

Source: Collection de l'artiste, 2016

À la manière de l'artiste de la terre Hamish Fulton, Urquijo n'intervient pas sur le lieu (le corps) parcouru. Elle se contente d'enregistrer ce qu'elle perçoit de sa vue attentive - une démarche qui laisse transpercer la sacralité qu'elle confère au corps. Comme pour Hamish Fulton, l'emplacement à franchir devient le site d'un rituel. Tous les deux mettent en rapport les photos prises lors de leurs promenades en y incorporant du texte, des signes et d'autres éléments. Pour Fulton, ce sont des « points 
de rencontre entre l'homme et la nature » (TIBERGHIEN, 2001, p. 102). Pour Lisette Urquijo, c'est une stratégie poétique qui guide notre regard et notre entendement. En scrutant de près chacun de ces découpages, ce sont au premier abord des morceaux isolés de tout, effectuant peu à peu une exploration vers les interstices territoriaux de l'intime, pour aboutir à toute une géographie du sujet. Chaque photo nous livre son détail distinctif, là où l'artiste a voulu nous faire pénétrer la singularité de son modèle.

Cette manière d'approcher le sujet et de le répandre en lambeaux photographiques rappelle la nouvelle vision des surréalistes. Avec l'apparition des appareils plus légers et en opposition au langage classique du siècle précédent, des artistes photographes des années 20 et 30 ont accentué « l'effet de fragmentation » (BAJAC, 2011 , p. 6) se focalisant sur le détail - voir par exemple le travail artistique de Helmar Lerski : la serie Verwandlungen des Lichts. Dans ce sens, Urquijo manie son appareil-cyclope comme ces surréalistes. D'autres artistes plus contemporains ont utilisé cette manière parcellaire d'aborder le corps, telle Sophie Ristelhueber, qui, par l'effet de démesure de ses grands tirages, convertissait les surfaces cutanées en matière visuelle. Mais dans Punto $G$, le rapprochement et l'encadrement de la prise de vue n'offrent pas au spectateur la possibilité de se perdre dans les précisions dermiques, ni de suivre méticuleusement chaque contour des plis, ou de rencontrer toutes les unicités spécifiques des surfaces cutanées telles les pores, les grains de la peau ou les taches, comme c'est le cas de Every One \# $8^{1}$ (1994) de Ristelhueber. En effet, cette œuvre d'Urquijo est constituée de petites photographies qui ne s'attardent pas dans les détails des pores rendus au contraire invisibles par le haut contraste qui baigne les photos. Cette œuvre nous offre une autre expérience visuelle, une sorte d'excursion cartographique.

Nous autres spectateurs, nous nous échappons ainsi avec l'artiste dans un voyage cutané, à la recherche semble-t-il d'un supposé point $G$. Nous sommes sensés inspecter un corps érotisé par le regard de l'artiste, et nous trouvons cet érotisme en effet dans certains clichés chargés de sensualité. Notre œil devient une loupe, un microscope scrutant la volupté du corps exposé, comme qui regarde une cartographie énigmatique d'une chasse au trésor, qui ici serait la zone érogène promise dans le titre de l'œuvre. Mais quelque chose d'autre vient interrompre ce dialogue avec l'érotisme. Certaines de ces photographies, telles celles de la médecine scientifique, sont d'une froideur impeccable. Elles rappellent également les prises utilisées dans le système d'identification criminelle par l'anthropométrie au XIXème siècle. Mais face à Point G, nous nous trouvons en présence d'un corps tout autant sensualisé que froidement médicalisé. Par là, l'artiste nous propose des chemins ouverts que l'on peut à notre guise survoler soit sur la cartographie cutanée présentée devant nous, soit dans notre imaginaire.

\footnotetext{
1 C'est une autre façon de parcourir le territoire corporel que nous montre l'œuvre de Sophie Ristelhueber, Every One \# 8 (1994), photographie en noir et blanc faisant partie d'une série où l'artiste affiche en très gros plans des fragments de visages chez des personnes venant d'être opérées. Les cicatrices ici présentes sont des signes qui attestent de la récente ouverture des corps. Ces traces sur la chair fonctionnent comme une écriture involontaire qui s'incarne sur la peau et témoignant ainsi d'histoires personnelles. Telle une carte topographique détaillant avec soin chaque recoin qu'elle contient, Every One \# 8 nous confronte à un espace cutané qui, par ses grandes dimensions, nous incite à nous y introduire. Par le changement d'échelle, l'artiste cherche à éveiller une hésitation chez le regardeur, afin qu'il se demande si ce qu'il voit est bien une « matière humaine. » Ici, le détail atteint par la fragmentation photographique débouche sur un terrain énorme dû à l'agrandissement, ce qui nous fait perdre tout repère avec la réalité. Le visage ainsi présenté dissimule son caractère humain. Avec son appareil, elle se dirige directement vers le signe corporel qu'elle choisi de photographier, pour une vue unique qui transforme le tout. C'est à notre tour en tant que spectateurs, de nous promener par le regard sur ce territoire laissé visible sur la surface photographique ; nous nous confrontons de fait à une peau étrangère contenant une mémoire particulière.
} 


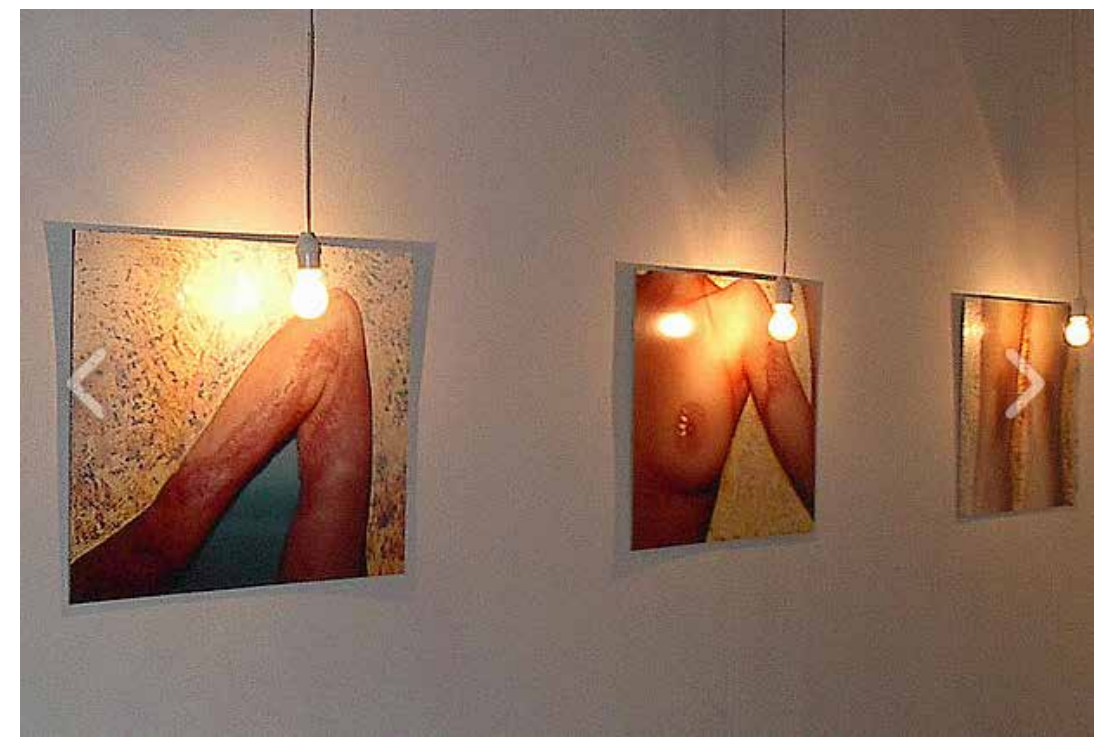

Fig. 2 - Lisette Urquijo, Inventario, 6 photographies argentiques, $50 \times 60 \mathrm{~cm}$ chaque image, 2003.

Source : <https://www.lisetteurquijo.com/>. Dernière consultation : 01/04/2018.

Inventario (2003) est le deuxième ensemble photographique de cette artiste que nous présentons ici. Six images argentiques en couleur de $50 \times 60 \mathrm{~cm}$ composent ce polyptyque représentant le corps d'une femme brûlée. À l'instar de Point G, Urquijo utilise ici une littérature du corps morcelée. Mais cette fois, des étendues plus grandes sont captées à la prise de vue. Les six photographies sont exposées de manière sobre, l'une suivant l'autre. Une source lumineuse est projetée, presque collée, sur chaque image : nous arrivons presque à sentir la chaleur envahissante des lampes. Face à nos yeux, ce corps nu, éclaté à la prise de vue, se recompose. Nous sommes à l'égard de cette œuvre sollicités à examiner les formes, tantôt géographiques, tantôt abstraites, des brûlures de cette femme, nageant tels des îlots sur un océan corporel.

La jeune femme ici portraiturée avait été mannequin dans son adolescence. Lors d'un tournage publicitaire, elle est accidentellement brûlée de manière grave. Malgré plusieurs interventions chirurgicales, l'ex-mannequin n'osait plus exhiber son corps, se rétractant dans une peau psychique de souffrance (ANZIEU, 1985). Cette collaboration entre l'artiste et son modèle a aidé la jeune femme à affronter sa honte et à montrer à nouveau son corps toujours beau et attirant. Encore une fois, cette œuvre nous offre un regard érotisé et en même temps médicalisé du corps. C'est un corps érotique car les formes sont pleines de rondeurs et de sensualité. C'est aussi un corps froid parce que le parcours qu'Urquijo trace marque des arrêts panoramiques, spécifiquement sur les blessures gravées à la superficie.

Au moyen de son intervention manuelle sur les photographies, Urquijo arrive à nous faire aller au-delà des empreintes déposées par le feu. Dans son laboratoire, l'artiste cherche à créer un jeu textural esthétisant, qui fasse écho aux marques laissées sur le corps de son modèle. De même au XIXème siècle, c'est grâce aux photographies esthétisées des malades que les lésions et les malformations sont devenues regardables et donc "observables ». Serge Tisseron raconte à cet égard que les malades provoquaient un dégoût visuel chez les médecins, rendant presque impossible de traiter les pathologies: «Faire de ces lésions des images qui puissent être regardées 
était la condition préalable à leur étude ». Ce travail sur les photographies cliniques a aidé à libérer le regard de la honte et de l'horreur " pour l'intéresser à la seule maladie » (TISSERON, 1999, p. 24). Aujourd'hui un corps comme celui d'Inventario est un corps désirable, malgré les traces laissées par le feu. À la différence de Point $G$, où l'artiste naviguait à sa guise, la traversée que Lisette Urquijo accomplit dans ce deuxième travail est guidée par ce que le corps lui-même a d'exceptionnel. Ainsi, ce sont ici les morsures laissées par le feu qui vont édicter les points spécifiques à cartographier.

Même si l'œuvre d'Urquijo est centrée sur la personne, elle contient déjà, on le sent bien, de fortes connotations sociales, tels les fantasmes individuels profondément irrigués par la société (point G), ou aussi bien dans l'implication d'autrui à travers son regard. Suite à ce parcours du corps ré-approprié qui résulte en un imaginaire érotique, nous analyserons maintenant des œuvres qui incarnent plus directement des positionnements face au politique.

\section{Territoires instables (AMOROCHO, 2018)}

Dans leur démarche les artistes présentées à partir de maintenant filtrent notre regard reliant le corps à des réalités sociales, telles que les conséquences de l'extractivisme ou, plus loin, les déplacements forcé, puis le féminicide.

Fréquemment, le corps de la femme et le territoire continuent à être comparés dans les combats féministes : outre des expositions ou des œuvres se focalisant sur ce sujet, des artistes prennent position, rapprochant métaphoriquement le corps féminin de la terre jadis conquise et prise de force. Un rapport très net entre l'urgence de la libération des corps et la délivrance des territoires sous l'influence des nouveaux conquérants que sont les multinationales se trouve, par exemple, dans les luttes des activistes s'opposant à l'« extractivismo ». Les groupes féministes qui se trouvent derrière ce combat assurent que la pensée patriarcale fait du corps de la femme ce que le marché « extractiviste » fait sur les territoires d'Amérique latine (GUILLAMON ; RUIZ, 2015). Plusieurs de ces organisations de femmes militantes, comme CASA ou RENAMAT, manifestent en défense de la terre, questionnant tant les modèles de développement actuels dans leur pays que les relations de pouvoir homme-femme, blanc-indigène, humain-nature. Selon elles, la femme dans le monde est confrontée à la soumission violente, spécialement s'il s'agit de pauvres et d'indigènes. Raison pour laquelle elles insistent sur le fait qu'il ne faut pas uniquement lutter pour la libération de la femme, mais qu'il est tout autant nécessaire de se battre conjointement pour la justice et l'égalité sociale. Ces féministes latino-américaines soutiennent que sous le modèle économique basé sur l'« extractivisme », la terre et les droits des femmes souffrent d'un affaiblissement : celles-ci y « vivent sous un harcèlement constant, il y a des violences sexuelles, des réseaux de traite de femmes [...] » (COLECTIVO CASA, 2015). Le point d'où nous sommes parties dans cette partie de notre étude a été ce parallèle entre la ré-appropriation du corps et la récupération du territoire.

Corps-nature et corps-politique sont ainsi des notions que l'on trouve dans le travail créatif d'artistes comme la Guatémaltèque Régina José Galindo. Lors d'une exposition au Chili, cette artiste réalisa Desierto (2011), une performance où elle resta allongée sur une table pendant deux heures. Elle recouvrit par ailleurs son corps de 
sciure, en référence directe aux problématiques liées à la désertification dans ce pays. Ainsi, Galindo pointe du doigt les conséquences qui découlent des abus commis aux dépens de la terre à cause des modes de culture. Elle affirme que la monoculture intensive du pin et de l'eucalyptus au Chili commence, déjà de façon visible, à ravager et à dessécher la terre. Outre les dégâts exercés actuellement sur l'écosystème, Galindo nous rappelle que l'histoire des territoires où se trouvent aujourd'hui les entreprises étrangères exploitant la terre est entachée de violence. En effet, sous le régime Pinochet, les Mapuches (communautés aborigènes qui habitent dans ces zones entre le Chili et l'Argentine) furent délogés de leur milieu par la violence et les massacres.

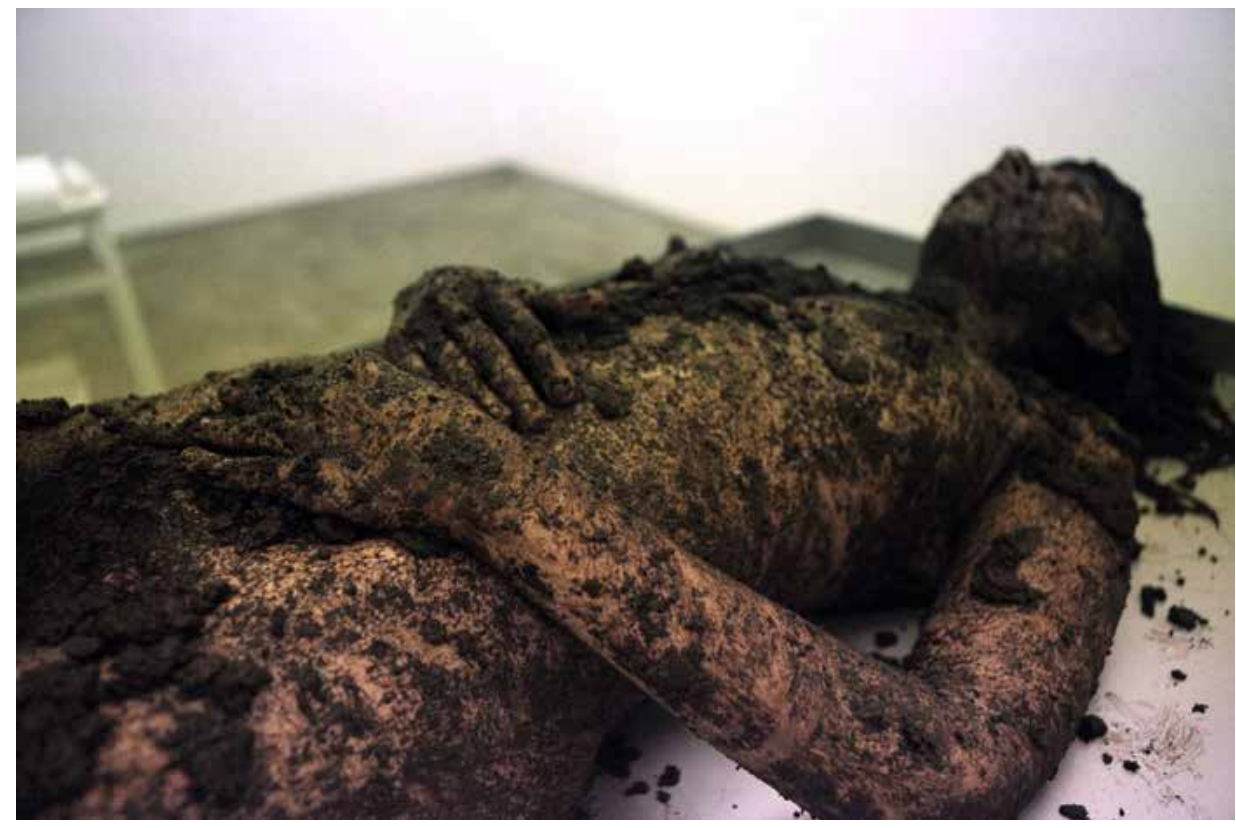

Fig. 3 - Regina José Galindo, Desierto, Performance, 2011

Source: <http://www.nodalcultura.am/2015/07/performance-artistica-que-une-geografias-e-historias-de-dictaduras/\#prettyPhoto>. Dernière consultation : 01/04/2018.

Cet épisode de l'histoire chilienne n'est pas un cas isolé, et en cela l'œuvre de Régina José Galindo est universelle car elle évoque aussi bien les oppressions dans d'autres latitudes du globe. Ainsi dans cette performance, le corps de la femme, embaumé de sciure de bois, évoque une sorte de paysage désertique privé de toute vie. Mort, violence et désolation, le contenu politique de cette œuvre de Galindo fonctionne en écho à des situations similaires advenues ailleurs. Par exemple au Guatemala, nous dit cette artiste, c'est la culture intensive de la palme africaine qui est au centre des catastrophes écologiques et sociales. Les populations se sont vues forcées de migrer vers les villes et, pour ceux qui restent, les conditions de travail et de vie sont extrêmement précaires. Quant à ceux qui osent réclamer des droits pour leur communauté, ils risquent de suivre le destin de l'activiste Rigoberto Lima qui fut assassiné. Face à ce constat, Galindo, sans cesse, veut dénoncer les atrocités commises sur les populations au Guatemala. Elle rappelle que pendant le conflit armé dans son pays, la stratégie de la « terre brûlée » fut utilisée par le gouvernement : « les cultures furent réduites en cendres, les femmes violées, les indigènes assassinés, les paysans expropriés »(GALINDO, in NODAL Culture, 2015). Terre brûlée, femme 
violée, peuples délogés, plus qu'une analogie, il s'agit d'une réalité qui est présente dans l'œuvre de cette artiste. Toute sa production créatrice est marquée par l'histoire de sa nation, en particulier relativement à tout ce qui touche aux violations des droits de l'homme.

\section{Déplacements forcés}

Les conflits armés internes qui s'abattent dans divers pays d'Amérique latine suscitent des exodes massifs, conséquence d'expropriations forcées et de massacres. En Colombie par exemple, les zones d'extractions de produits naturels, tels l'or, hébergent des nœuds de violence. L'histoire récente de ce pays montre de nombreuses atrocités commises dans ses régions convoitées, induisant des migrations non-voulues vers les capitales régionales. Les corps déplacés par le conflit sont ce que l'artiste colombienne Libia Posada photographie dans Signos cardinales (2008). Une série d'images en noir et blanc où l'on peut apprécier la partie inférieure de jambes féminines sur lesquelles figurent des cartes mémorielles sous forme de tatouage. Plus qu'une géographie territoriale, ces dessins éphémères sur la peau indiquent une cartographie imagée de la souffrance, de la fuite, des périples et des repères que ces personnes déplacées ont gardés en mémoire.

L'encre noire estampée sur ces jambes dans Signos cardinales décrit les voyages sans retour endurés par onze individus. Dès leur arrivée à Medellín, l'artiste a rencontré ces femmes, qui, à sa demande, lui ont détaillé le mieux possible le parcours emprunté, faisant appel à leurs souvenirs douloureusement infusés de peur et de mélancolie. Posada reconstruit à partir de ces récits des lignes synthétiques figurant l'histoire dramatique de leur fuite ; et ceci à travers ces cartes mentales.

Quand on voyage, on détermine auparavant une route à suivre, signalant tant le point d'arrivée et de départ que les endroits à traverser. Partir sous la contrainte implique pourtant d'inaugurer des chemins ou de parcourir des trajets dans l'urgence, difficiles à localiser, à reconnaître, à comprendre, et à voir, non seulement dans la mémoire de ceux qui fuient, mais aussi dans cette représentation du territoire dénommée carte (GALLÓN SALAZAR, 2010. Traduction personnelle).

explique l'artiste pour qui le corps fonde le territoire le plus précieux pour l'être humain.

Ces cartographies de récits sont peuplées de signes qui expliquent, entre autres, comment ces endroits furent traversés et vécus émotionnellement. Ainsi, nous voyons dans les photographies des lignes en pointillé, des croix et d'autres signes annonçant aussi bien des petits chemins, des zones de massacres ou des routes minées ; d'autres symboles encore sont figurés pour signaler les églises, les rivières, les reliefs, etc. Les photos qui documentent ces cartes temporaires ne fonctionnent pas uniquement comme des archives statiques qui nous permettent de percevoir les processus de remémoration de ces femmes, mais elles sont en soi une trace physique dans le présent et nous rappellent ce qu'on voit tous les jours dans les médias sans plus y faire attention. Comme si le temps marqué par les massacres et la violence en général aurait un effet anesthésiant sur les populations. Or, cette œuvre nous enlève cette 
morphine de l'accoutumance et nous montre les conséquences de la géopolitique économique incarnée dans les corps-territoires de ces onze personnes.

\section{Féminicide : frontières d'un autre genre}

La violence sexuelle et les conflits armés sont devenus communément associés². Des écrivaines théorisant sur le féminicide telles que Marcela Lagarde y de los Ríos (2008) ou Rosa-Linda Fregoso (2010) font également le rapprochement entre ces deux aspects de la violence. Pendant les génocides des années 90 en Bosnie et au Rwanda, le viol fut utilisé comme stratégie militaire afin de construire une nouvelle nation dans le cas des paramilitaires Serbes et de nettoyer le pays pour les Hutus dans l'État africain (MAY SCHOTT, 2010, p. 25). Ouvertement dénoncée par la communauté internationale, cette manière de procéder fut largement médiatisée. Grâce aux médias ainsi qu'aux débats et aux luttes féministes, depuis 2002 cet usage est considéré par la Cour Pénale Internationale (CPI) comme crime de guerre et crime contre l'humanité3.

Du côté de l'Amérique latine, des dirigeants politiques ont mis en pratique cette méthode afin de terroriser leur population. Ce fut le cas, par exemple, du Salvador, du Pérou et de Haïti, pendant leurs guerres civiles. Les dictatures en Argentine et au Chili ont instauré ce procédé dans les camps de torture. Au Guatemala, pendant la longue période de guerre civile, les forces de l'État ont torturé et violé des femmes indigènes de tous âges, jusqu'à l'avortement suivi de leur mort pour celles enceintes. Plusieurs artistes ont développé des œuvres concernant ce sujet comme Janet Toro qui, à partir d'entretiens avec des survivantes chiliennes prisonnières pendant la dictature Pinochet, a répertorié 60 méthodes de torture et réalisa une série de 90 performances et d'installations sous le titre El cuerpo de la memoria.

Pendant les années 90, au Mexique, des formes « indescriptibles de dégradation, de viol » (FREGOSO, R. L. ; BEJARANO, 2010, p. 3), de torture et de mutilation, avec un acharnement particulier sur les parties anatomiquement féminines, sont trouvées sur les cadavres de jeunes femmes abandonnées dans le désert à la frontière de Juarez. En raison du nombre important de corps retrouvés, des féministes ont commencé à nommer la situation fémicide ou féminicide 4 . Un débat existe sur le terme à appliquer, mais celui qui est resté en Amérique latine et en France est celui de « féminicide ». Ce concept a aujourd'hui traversé les frontières du Mexique. En France, le mouvement Osez le féminisme a lancé une campagne en 2014 pour la reconnaissance légale du féminicide. Selon ce groupe, "tous les deux jours et demi, une femme est tuée par son conjoint ou ex-conjoint ». Ainsi, le néologisme dénonce les meurtres de «

\footnotetext{
2 Les crimes sexuels sont perpétrés contre des femmes civiles partout dans le monde, et plutôt que de diminuer, le nombre de ces crimes augmentent effroyablement. Amnesty International rapporte que quarante mille femmes et filles furent violées au Congo dans la période 1999-2005. La torture sexuelle a été documentée au Rwanda, au Soudan, en Yougoslavie, en Irak et au Timor oriental » (BOOTH, 2005, p. 118). Traduction personnelle. Cité dans: MAY SCHOTT, R., 2010, p. 49. 3 En application de cette loi, la Cour Pénale Internationale a condamné en 2016 l'ex-vice-président congolais Jean-Pierre Bemba, à 18 ans de prison pour les crimes commis par ses troupes dans la république centre-africaine entre 2002 et 2003. (MAUPAS. 2016).

4 La sociologue Julia Monarrez Fragoso a utilisé le terme féminicide pour décrire les assassinats sexuels des femmes et des filles observés en 1993 par Ester Chavez Cano à la frontière entre le Mexique et les États-Unis à Juarez. Le mot avait déjà été employé dans les années 80 en Amérique latine. Par ce vocable sont désignés les meurtres de femmes dans des contextes en dehors de la guerre et les meurtres systématiques pendant un conflit comme celui de Bosnie-Herzegovine. L'appellation a été adoptée depuis dans des réunions des Organisations Non Gouvernementales (ONG) pour sensibiliser le public à ce problème. C'est en 2007 que La Court Américaine des Droits de l'Homme s'est servie pour la première fois du terme lors du procès international concernant les assassinats du Campos d'Algodón. Alice Laurel Driver résume l'histoire du terme comme suit : « féminicide » fut inventé en 1801 en Angleterre et rendu populaire en 1975 quand l'activiste Sud Africaine Diana E.H. Russell publie son livre The Politics of Rape: The Victims Perspective et en 1992 dans Femicide: The Politics of Woman Killing.
} 
femmes » dans une perspective de genre qui cherche à rompre avec « les notions essentialistes de l'identité féminine [et qui] regarde plutôt la nature genrée des pratiques et des comportements » (FREGOSO ; BEJARANO, 2010, p. 3). Cette forme d'assassinat ne survient pas uniquement pendant les guerres : il est présent aussi durant l'après-guerre et les périodes de paix. Elle ne s'exécute pas exclusivement en masse ni systématiquement. Elle se passe aussi dans des situations privées et quotidiennes, voire elle est exercée par des proches. Pour Rosa-Linda Fregoso et Cynthia Bejarano (2010, p. 4), arriver au concept de féminicide leur a permis de « cartographier les dynamiques de pouvoir dans les relations des sexes et de race aussi bien qu'entre les classes sociales ", en mettant l'accent sur la violence qui y est implicite. Ainsi, disent-elles, sous le joug de ce dispositif de pouvoir fondé sur des inégalités, les femmes deviennent automatiquement les cibles préférentielles de la violence, tant dans le domaine public que privé. Le cas de Ciudad Juárez a été un moteur de révolte et de création pour une multiplicité d'artistes de dimension nationale (Méxique) et internationale dans tous les domaines : arts plastiques, cinéma, théâtre, littérature...

Nous parlerons maintenant de deux œuvres d'artistes mexicaines touchant le thème du féminicide, et réalisées sous une forme « cartographique »: Desaparecidas (Disparues) de Maya Goded réalisée en 2005-2006 et Mientras dormiamos (el caso Juárez) (Pendant que nous dormions [le cas Juarez]) de Lorena Wolffer (2002-2004). Maya Goded photographie les emplacements où l'on a trouvé les corps meurtris, les endroits où les filles ont été enlevées, les familles des victimes marquées par la douleur, l'espace qui avait été le leur, etc. Il s'agit d'une cartographie de l'absence. En regardant ces photos, l'on peut constater la provenance sociale des jeunes filles abattues : dans la majorité des cas, des pauvres. Les environnements exposés montrent des coins géographiquement marginaux qui forment le bord des villes, et dont les carences en infrastructures urbaines sont frappantes; les personnes s'y installent par contrainte économique. Pour la réalisation de cette œuvre, et avec l'intention de connaître en profondeur la réalité de la situation, Goded a travaillé en collaboration avec la Commission des Droits de l'Homme du District fédéral de la ville de Mexico. Elle a écouté les histoires déchirantes rapportées par les familles lors de leurs rencontres. Cette série d'images a été pour l'artiste « la chose la plus difficile qu'elle a pu photographier » (CASTILLO, 2011) et pas uniquement à cause des difficultés rencontrées sur place, comme le vol de ses appareils, la maladie qui l'a clouée au lit ou la tentative d'enlèvement dans sa chambre d'hôtel. Cartographier l'absence, c'est parler de quelqu'un qui n'est plus là et dont nous pouvons pourtant respirer la présence. Les images de Desaparecidas, notamment celles des chambres, des lieux de fréquentation, des lettres ou des affaires personnelles des disparues, nous permettent de voir ou de reconstruire dans notre imaginaire qui étaient ces filles. Ces photos nous montrent des âmes en permettant de donner un visage et une personnalité aux corps absents. Par celles qui révèlent l'école d'où une fille à été enlevée, ou la place de Lomas de Poleo où un corps a été retrouvé, par exemple, nous sommes en mesure de retracer certains points du calvaire vécu par ces femmes avant de devenir des cadavres. Ainsi, cette œuvre de Maya Goded a une très forte charge émotionnelle. 


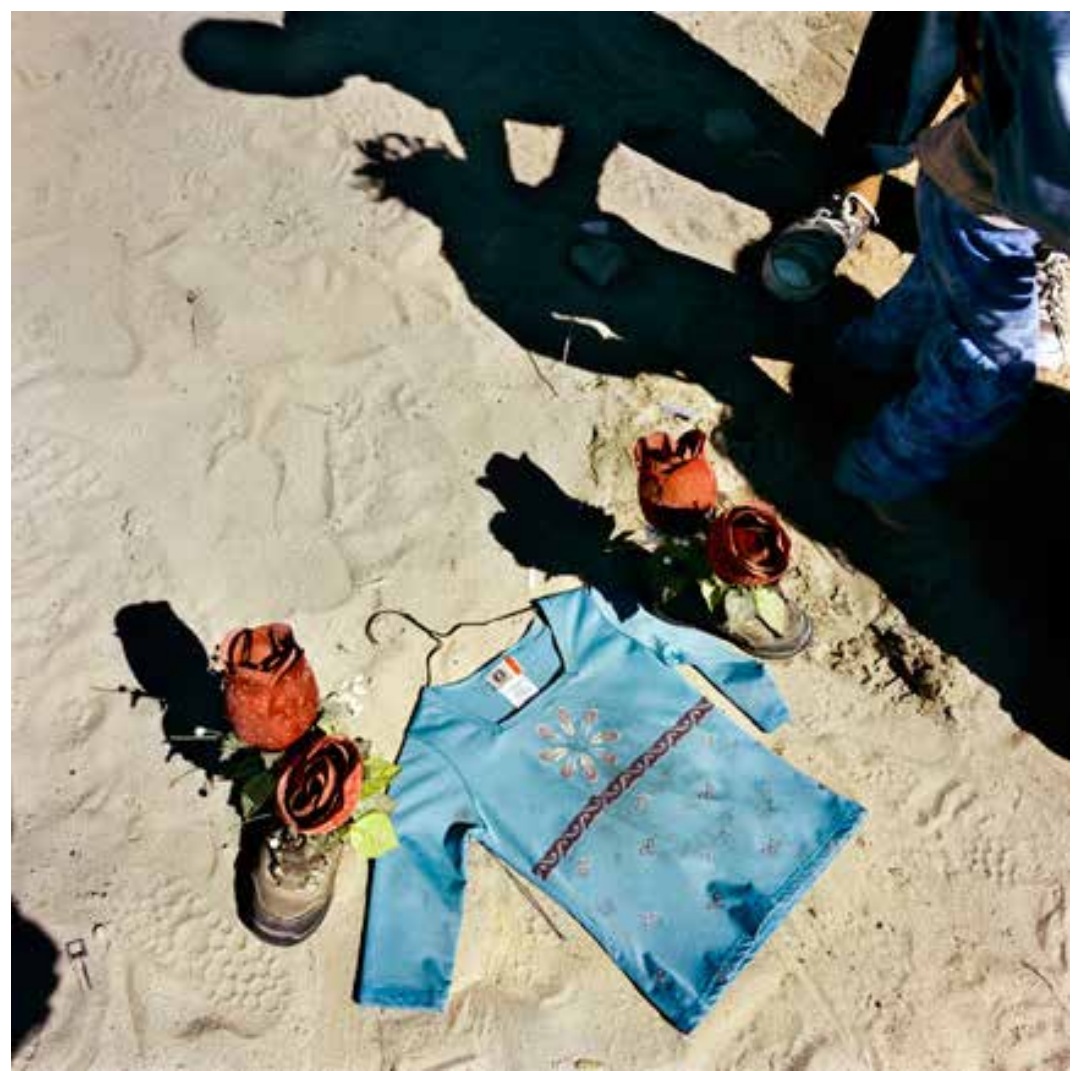

Fig. 4 - Maya Goded, Desaparecidas, Photographie (polyptyque), 2005-2006.

Source : <https://www.animalpolitico.com/2011/08/la-fotografia-acompana-mi-soledad/>. Dernière consultation : 01/04/2018.

L'impunité qui couvre les centaines de meurtres de femmes à Ciudad Juarez indigne vivement l'artiste. De plus, un discours sexiste accompagne ces féminicides: les femmes sont accusées « d'habiter l'espace public, de marcher seules et de sortir le soir » écrit Laurel Driver (2011, p. 6) dans sa thèse. En effet, dans une société marquée par le machisme, les femmes sont confinées à des rôles sociaux stricts qui définissent leurs conduites, jusqu'aux horaires de sortie et aux vêtements appropriés à l'espace public. Les filles retrouvées mortes « l'ont cherché » selon certains, à commencer par le gouverneur de l'État de Mexico Eruviel Ávila, qui à ce sujet a ouvertement exprimé son désintérêt : "[...] il y a des choses plus graves desquelles se soucier ${ }^{5}$ ». La série de 12 photographies en couleur tirées à grande échelle, qui rend hommage aux absentes, a participé à diverses expositions consacrées au féminicide, comme Frontera +450 (2006-2007) et Ni una Más: the Juárez murders (2010).

5 Réponse donnée à l'Observatorio Ciudadano Nacional del Feminicidio (OCNF). 


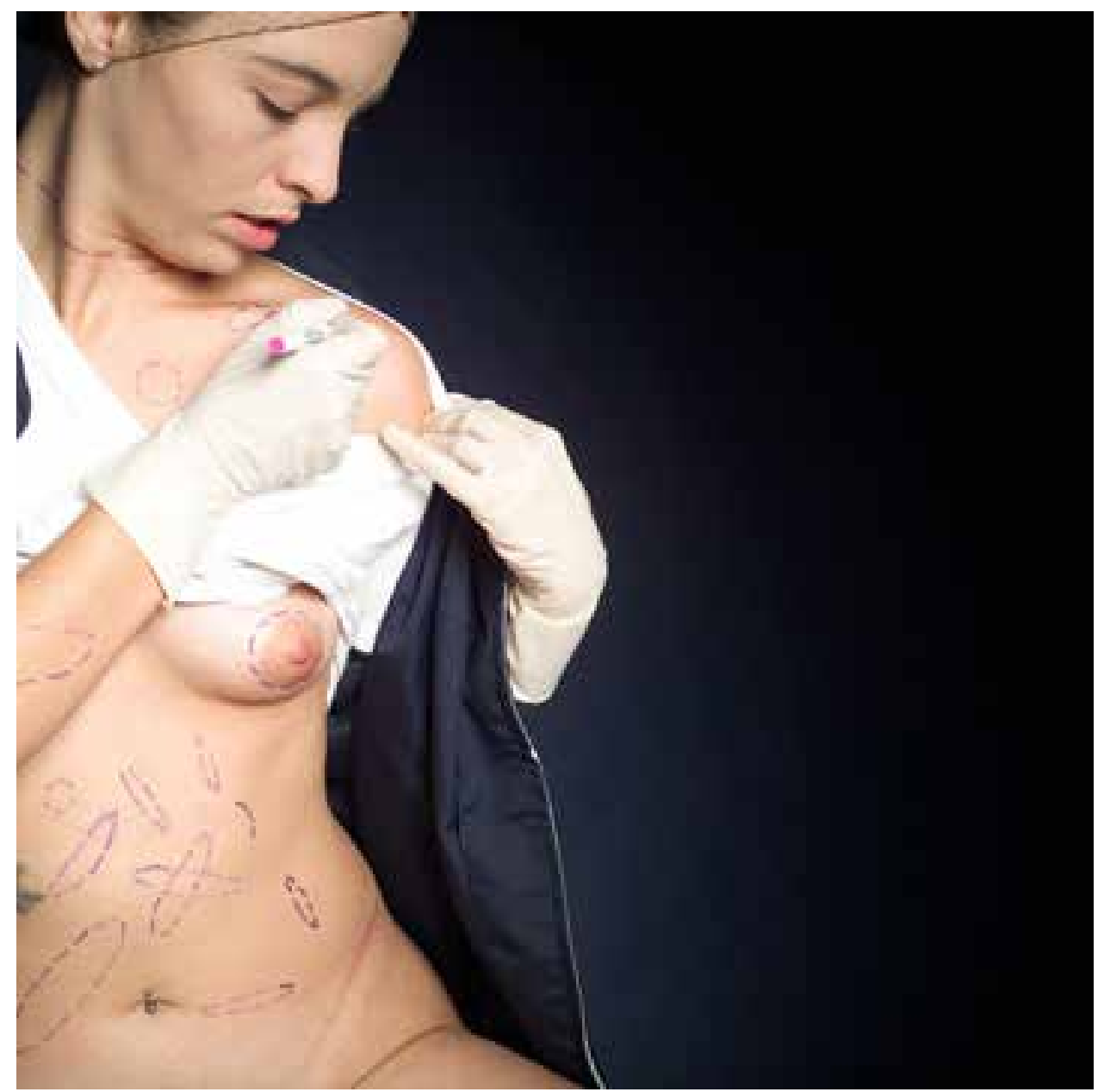

Fig. 5 - Lorena Wolffer, Mientras dormíamos (el caso Juárez). Performance (photographies : Martín L. Vargas), 2002-2004. Source : <http:/l www.lorenawolffer.net/010bra/11md/md_frames.html>. Dernière consultation : 01/04/2018.

Mientras dormiamos (el caso de Juarez), deuxième œuvre dont nous voulons parler à propos du féminicide, est une performance où l'artiste Lorena Wolffer va tracer une cartographie de cette problématique sur la surface de son corps. Tout au long de l'action, après une brève sonorisation de cris de femmes, nous entendons une voix off masculine réciter des documents policiers décrivant froidement l'état d'une cinquantaine de corps trouvés à Juárez. Ce faisant, l'artiste procède à marquer sur sa peau, à l'aide d'un stylo chirurgical, chaque blessure, tir de balle, coupure, mutilation, égorgement, etc. décrits dans le document acoustique. À la fin de la performance, son corps est quasiment englouti dans l'encre. Il disparaît sous les traits d'une carte funeste devenant un territoire presque conceptuel. Sorte de " sanctuaire temporel », comme dirait Guillermo Gómez-Peña (2005, p. 23), le corps ainsi cartographié pendant la performance nous met face à la réalité troublante du féminicide. Pour Lorena Wolffer, le corps est le lieu privilégié de la contestation politique. Dans le cas de Mientras dormiamos, son intention est de divulguer l'information sur ces atrocités commises dans ce district frontalier. Les noms et prénoms sont énoncés, humanisant par là les victimes, alors que dans la réalité, seul un numéro leur est assigné.

Chaque détail est finement étudié par Wolffer. Au début de son action, l'artiste est couchée sur une table type morgue ; elle porte l'uniforme utilisé dans les maquiladoras, nom générique donné aux entreprises d'assemblage états-uniens installées au Mexique depuis 1965 grâce au Programme d'Industrialisation de la Frontière (BIP) 
signé entre les deux nations (WOOD, 2008, p. 17). Les maquiladoras emploient des milliers de femmes, forcées à travailler sans garanties des heures interminables en contrepartie d'un maigre salaire et dans des conditions précaires et dangereuses. Finissant très tard, en raison de l'éloignement des entreprises et du manque d'infrastructures publiques dans les transports et l'électricité, elles doivent attendre longtemps et dans l'obscurité le bus qui les ramènera chez elles. C'est dans ces circonstances que plusieurs de ces travailleuses ont été enlevées puis ont trouvé la mort dans des conditions dramatiques. Face à cela, les autorités font preuve d'une négligence certaine. Cet aspect est pris en compte par Lorena Wolffer qui, à la fin de sa performance, se cache entièrement sous des couvertures, à la manière d'un cadavre, dénonçant par là « l'institutionnalisation de ces crimes et la passivité de la justice mexicaine devant ces faits » (BALLESTER, 2012, p. 11).

\section{Conclusion}

À travers ce parcours succinct, sillonnant des œuvres plasticiennes qui centrent leur discours sur le corps, nous avons établi un rapprochement avec l'écriture cartographique. Cette écriture s'effectue soit par le geste de traverser le corps tout en le fragmentant ou de le déchiffrer dans le cas des travaux étudiés dans la première partie, soit par l'utilisation du corps comme support d'une représentation abstraite tels les exemples abordés dans les parties suivantes. Créant une écriture, une réécriture, un brouillon ou de la poésie, les œuvres ici analysées arrivent à composer avant tout des phrases transgressives, qui parviennent à transformer la matérialité du corps en signe, pouvant de cette manière en décrire les paysages intimes, en ériger des cartes territoriales ou en déjouer les frontières. Par leur positionnement, ces artistes font de l'image une question de prise de parole contre la violence, le féminicide, l'extractivisme et la domination en vue d'une ré-appropriation des corps. Ce sont des promenades vertigineuses qu'elles nous font découvrir, qui mettent l'accent sur le présent d'une réalité sociale qu'elles nourrissent de leurs motivations politiques, éthiques et personnelles.

\section{Bibliographie}

AMOROCHO, M. Aux confins de la chair. Esthétique de l'irreprésentable. Ou la mémoire traumatique face aux violences sexuelles. Édition bilingue espagnol-français. Bogota : Apidama Ediciones Ltda, 2018.

ANZIEU, D. Le Moi-peau. (Nouvelle édition) Paris : Dunod, coll. : « Psychismes $», 2004$.

BAJAC, Q. Le corps en éclats. Paris : Éditions du Centre Pompidou, coll. : « La collection de photographies », 2011.

BALLESTER, I. « Métaforas extremas ante el dolor y desde el feminismo ", in Dossiers féministes, 16, 2012. Disponible sur: <https://dialnet.unirioja.es/descarga/ 
articulo/4235281.pdf>. Dernière consultation : 31/03/2018.

CASTILLO, M. La fotografía acompaña mi soledad. Méxique: Animal Político, 13/08/2011. Disponible sur: <http://www.animalpolitico.com/2011/08/la-fotografia-acompana-mi-soledad/>. Article consulté le 05/09/17.

COLECTIVO CASA. Mujeres feministas articulan lucha conjunta frente al extractivismo. 11/03/2015. Disponible sur: <http://www.colectivocasa.org.bo/index. php?option=com_k2\&view=item 8 id=183: mujeres-feministas-articulan-lucha-conjunta-frente-al-extractivismo>. Dernière consultation : 31/03/2018.

CRUZ HERNÁNDEZ, D. T. « Una mirada muy otra a los territorios-cuerpos femeninos » in Solar, Año 12, Volumen 1, Número 1, Lima, 2016. Disponible sur: <http://revistasolar.org/wp-content/uploads/2017/07/3-Una-mirada-muy-otra-a-los-territorios-Cuerpos-femeninos.-Delmy-Tania-Cruz-Hernández.pdf>. Dernière consultation: 31/03/2018.

DRIVER, A. L. Cultural production and ephemeral art: feminicide and the geography of memory in ciudad juárez, 1998-2008, Thèse Doctorale d'études hispaniques, University of Kentucky, 2011. Disponible sur: <https://uknowledge.uky. edu/cgi/viewcontent.cgi?article $=1001 \&$ context=hisp_etds $>$. Dernière consultation: 31/03/2018.

FREGOSO, R-L. et BEJARANO, C. Terrorizing Women. Feminicide in the Américas. Londres: Duke University Press, 2010.

GALLÓN SALAZAR, A. Mi cuerpo es mi territorio. Bogota : El espectador, 20/12/2010. Disponible sur: <https://www.elespectador.com/jscroll_view_entity/ node/241530/full/p243412shown>. Dernière consultation: 31/03/2018.

GARTOR, M. El feminismo reactiva la lucha contra el "extravismo" en América Latina. Espagne: La Marea, 17/02/2014. Disponible sur: <http://www. lamarea.com/2014/02/17/ecuador-extractivismo-mujeres/>. Dernière consultation: $31 / 03 / 2018$.

GÓMEZ-PEÑA, G. Ethno-Techno: Writings on Performance, Activism and Pedagogy. New York et Londres: Routledge, 2005.

GUILLAMON, A. ; RUIZ, C. « Feminismos y lucha por el territorio en América Latina ». in Pueblos-Revista de información y debate, $N^{\circ} 64,09 / 02 / 2015$. Disponible sur: <http://www.revistapueblos.org/blog/2015/02/09/feminismos-y-lucha-por-el-territorio-en-america-latina/>. Dernière consultation: 31/03/2018.

KRISTEVA, J. Sens et non-sens de la révolte (Discours direct): Pouvoirs et limites de la psychanalyse I. Paris: Fayard, 1996. 
LAGARDE Y DE LOS RÍOS, M. « Antropología, feminismo y política. Violencia feminicida y derechos humanos de las mujeres », in: Retos teóricos y nuevas prácticas, sous la dir. de BULLEN, M. L. ; DÍEZ MINTEGUI, M. C. Espagne : Ankulegi, Publié lors du Congres d'Anthropologie, 11. 2008. San Sebatián. Disponible sur: <https:// www.ankulegi.org/wp-content/uploads/2012/03/0008Lagarde.pdf>. Dernière consultation: 31/03/2018.

LIPPARD, L. Overlay. Contemporary Art and the Art of Prehistory. New York: Pantheon Books, 1983.

MAUPAS, S. Jean-Pierre Bemba jugé coupable de crimes contre l'humanité en Centrafrique, France: Le monde, 21/03/2016. Disponible sur: <http://www. lemonde.fr/afrique/article/2016/03/21/l-ancien-vice-president-congolais-juge-coupable-de-crimes-contre-l-humanite-en-centrafrique_4887192_3212.html>. Dernière consultation: 31/03/2018.

MAY SCHOTT, R. (dir.), Birth, Death and femininity. Philosophies of Embodiment, Bloomington et Indianapolis: Indiana University Press, 2010.

TIBERGHIEN, G-A. Nature, Art, Paysage, Paris: Actes Sud Nature, Hors collection, 2001.

TISSERON, S. Le mystère de la chambre claire: Photographie et inconscient, Paris : Flammarion, coll. « Champs », no 443 », 1999.

WOOD, O. An Investigation into Exploitation of the Mexican Female Body along the U.S.-Mexico Border, Honours American and Canadian Studies, 2008. (Le pdf de cette recherche n'est plus disponible sur internet en libre accès). Consulté le 27/03/15. 